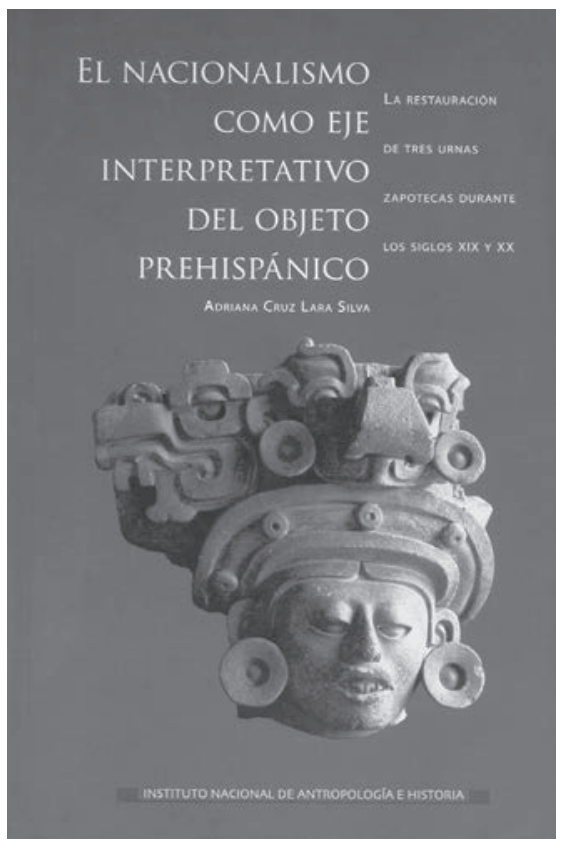

Portada del libro de Adriana Cruz Lara Silva El nacionalismo como eje interpretativo del objeto prehispánico. La restauración de tres urnas zapotecas de los siglos XIX y XX, México, INAH, 2011.

\section{El nacionalismo como eje interpretativo del objeto prehispánico: en busca del origen y los motivos que condujeron a la restauración masiva de tres urnas oaxaqueñas excavadas durante el régimen porfirista}

\author{
Laura Filloy Nadal
}

E I libro que hoy nos ocupa, El nacionalismo como eje interpretativo del objeto prehispánico. La restauración de tres urnas zapotecas de los siglos XIX y XX, de Adriana Cruz Lara Silva, cerró oportunamente el año 2011. Publicado por el Instituto Nacional de Antropología e Historia (INAH) dentro de la colección dedicada a la Conservación y Restauración del Patrimonio (serie Fundamentos), en un formato de pequeñas dimensiones, nos permite disfrutar de una sola sentada sus 110 páginas. Está dividido en cinco capítulos e incluye una atinada bibliografía que da cuenta de la variedad de temas que en el libro se revelan.

Adriana Cruz Lara Silva es restauradora e historiadora del arte, áreas del conocimiento que revisa, critica y entrelaza a lo largo de su texto. Durante el verano de 1999 colaboró en el Proyecto Integral de Reestructuración del Museo Nacional de Antropología (PIR-MNA), en cuya primera etapa se restauraron objetos pertenecientes a las culturas mixteca y zapoteca de la sala Oaxaca de dicho museo. Fue precisamente durante esa experiencia cuando, gracias a la observación y al trabajo directo con las piezas, los restauradores del PIR-MNA constataron que un sinnúmero de objetos presentaban intervenciones de diversa índole, entre las que destacaba la reconstrucción de grandes secciones en ciertas piezas de cerámica, las cuales habían pasado inadvertidas incluso para el personal que laboraba en las distintas áreas del museo desde hacía mucho tiempo, así como para diversos estudiosos del arte prehispánico que tuvieron la oportunidad de analizarlas. Como las áreas reconstruidas se confundían fácilmente con las secciones originales, en aquel momento se hizo patente la intención de quienes las habían realizado: evitar a toda costa que fueran evidentes. Aunque el grupo de restauradores del PIR-MNA pronto se interesó en conocer la época y la autoría de dichas intervenciones, la premura del proyecto impidió llevar a cabo los estudios necesarios. Al concebir su libro, Adriana Cruz Lara retomó y amplió dichas interrogantes; para iniciar la discusión se pregunta: "¿en qué época se restauraron las piezas y quiénes las hicieron? ¿A qué visión nos remite la manera como fueron intervenidas? ¿Qué 
debemos hacer frente a estas intervenciones históricas? ¿Qué significado tienen para las áreas dedicadas a su estudio, como la historia del arte, la arqueología y la restauración?" (Cruz Lara 2011: 14). Con el fin de responderlas, seleccionó tres urnas de cerámica pertenecientes a la cultura zapoteca de la colección del MNA: la "Dama 13 Serpiente" (Inventario 10-3260); una urna procedente de Ocotlán, Oaxaca (Inventario 1079927), y otra con la representación del dios Pitao Cozobi (mencionada en las fichas catalográficas del museo como "Urna de Pitao Xoo").

En el primer capítulo Cruz Lara establece una comparación entre las pautas que siguió la arqueología oficial durante los 1800s y las que observó la restauración de edificios prehispánicos en el curso del siglo $X X$, con el objetivo tanto de ubicar al lector como de evaluar si dichos cánones, reflejo de la ideología nacionalista que imperaba en México, se emulaban al intervenir los objetos de cerámica. Coincido con la autora en que la acción de restaurar, el cómo, sus alcances, las perspectivas teóricas y metodológicas en las que se sustenta la intervención son reflejo directo de la época y de la ideología vigente al momento de intervenir una pieza. Por su parte, Cruz Lara (2011: 25) menciona asimismo que ello abonará en la "construcción de un nuevo objeto", "más complejo, mayormente rico y elocuente en cuanto a la historia que tiene para contarnos", y que a través de la restauración adquiere nuevos valores que inciden en su forma y significación.

En el segundo capítulo la autora hace un breve análisis de la función que tuvieron las urnas en la cultura zapoteca: el culto a los ancestros practicado por la elite, y en pocas páginas discute el contexto histórico de su creación, así como sus cualidades estéticas y los distintos trabajos académicos que se han ocupado de ellas. "El hallazgo de una restauración desconocida", título del siguiente capítulo, refleja el móvil ori- ginal y detonante de su trabajo: en él enumera los objetivos principales del PIR-MNA y sus alcances, y continúa con la descripción de las tres urnas que constituyen el corpus de su estudio, para concluir con los pormenores de las características de las viejas intervenciones, reveladas en los albores del siglo XXI. En este último tema Cruz Lara esboza algunas críticas acerca de la inconsistencia en la toma de decisiones, por parte del PIR-MNA, para solucionar las lagunas de gran tamaño o faltantes de dimensiones considerables. Me habría gustado que hubiese profundizado en el análisis de las perspectivas teóricas y metodológicas utilizadas por los restauradores del proyecto, cuyas decisiones modificaron para siempre la valoración que se tenía del objeto y que desde 1999 forman parte de su biografía más reciente, un juicio que seguramente sería de utilidad para todos aquellos que trabajamos en el MNA.

Para terminar el capítulo tres, la autora desarrolla su hipótesis de trabajo, y concluye que el origen y los motivos que dieron lugar a la reconstrucción masiva de las urnas fueron las políticas nacionalistas del Porfiriato, las cuales alentaron el coleccionismo de piezas arqueológicas en tanto que reflejaban, por un lado, la grandiosidad del pasado prehispánico de México y, por el otro, el gusto refinado de la clase en el poder.

En el capítulo cuarto, que mucho despertó mi interés, la autora se interroga continuamente, discute $y$ construye, de manera sencilla y bien argumentada, aquello que le preocupa: los criterios con los que se completaron las urnas. La trama se desarrolla de manera fluida y va llevando al lector a comprender el momento histórico en el cual se realizaron las restauraciones $y$, al final, las corrientes vigentes en México al concluir el siglo XIX y principios del $x x$-emuladoras de lo que ocurría en otros museos del mundo-, que dieron lugar tanto a la excavación de sitios prehispánicos como al coleccionismo de objetos selectos y su posterior ingreso en el Museo Nacional, entidad que reflejaba los ideales del régimen porfirista que estudiaba, conservaba y difundía las glorias del pasado.

El tránsito continuo entre el momento en que las piezas se observaron por primera vez desprovistas de sus reconstrucciones en el año de 1999 y los albores del primer centenario de nuestra independencia, permite que el lector consiga la información necesaria para comprender la historia reciente de estos tres objetos, su inclusión o exclusión de las salas de exhibición del viejo y el nuevo museo, así como su ubicación actual en el discurso museográfico del renovado MNA.

Uno de los hilos conductores de este capítulo lo constituye el análisis de los distintos actores sociales que animaron la restauración original de los objetos, y de cómo los interpretaron en primera instancia y los emplearon como propaganda de la estética oficial del México prerrevolucionario. Cruz Lara rastrea las razones que movieron a tres coleccionistas oaxaqueños, que pertenecían al denominado Club Arqueológico, a explorar y excavar en la región de Oaxaca, lo que probablemente derivó en el hallazgo de las tres urnas en cuestión. Por su parte, tal y como lo narra la autora, Francisco Belmar y Fernando Soleguren jugaron un papel determinante para que las urnas ingresaran al museo. Seguro ellos determinaron, a través de una postura estética definida, la imagen final, restaurada y unitaria, que conocimos en 1999. Sus motivaciones y acciones apenas comienzan a vislumbrarse.

El quinto capítulo reflexiona sobre la musealización de las urnas en siglo $\mathrm{xx}$, primero en el viejo edificio de la calle de Moneda y posteriormente en el nuevo, del Bosque de Chapultepec (antes y después de la reestructuración de las salas). Aquí se describen las formas de agrupación de los objetos, la primera seguida conforme a criterios estilísticos o de forma, para dar paso, a partir de 1964, a una disposición de índole 
cronológica. Este apartado discute la posición que ha tenido el museo en las políticas culturales del México moderno y resuelve, como otros autores que la precedieron, que a lo largo de sus dos siglos de existencia dicha institución ha sido un actor activo de la política nacionalista de los distintos gobiernos, que buscaron, sin lugar a dudas, crear una identidad clara y distinta, basada en su raíz indígena, para el México moderno.

A manera de conclusión, y después de hacer un recorrido sobre los distintos temas tratados en el libro, Adriana Cruz Lara propone la revisión del criterio con el que actualmente se exhiben las urnas en el $M N A$, donde: se incluya "todo lo que han representado, los procesos de los que han formado parte, las ideas y sentimientos que han propiciado" (Cruz Lara 2011: 102); se informe al visitante de la historia reciente del objeto y de sus distintas restauraciones, y se resalte el valor inherente de cada uno de ellos.

Indudablemente, el estudio del significado de las restauraciones históricas en el contexto que las originó, y la información que proporcionan sobre la historia reciente del objeto, son un campo poco explorado en México. Así, el análisis de Cruz Lara sobre estas tres piezas debería haberse extendido al conjunto de urnas zapotecas que ingresaron en las colecciones del INAH en la primera mitad del siglo xx. Ello habría propiciado una mejor comprensión del desarrollo que ha tenido la restauración de piezas cerámicas en México y de los conceptos teóricos en los que se basó cada intervención, así como una profundización en la postura estética de los coleccionistas, donde, a decir de la autora, "lo unitario se contrapone a lo fragmentario", y cada una de ellas se hizo:

[...] para complementar las áreas faltantes de las piezas, reproduciendo formas y atributos que les permitan adquirir una apariencia completa y una posibilidad de lectura sin interrupciones, priorizándose la apariencia visual sobre cualquier otra consideración. El valor arqueológico de los objetos, en tanto testimonios de las formas de vida de la civilización zapoteca, pasó a un segundo término para favorecer su presentación estética (como un objeto completo)" (Cruz Lara 2011: 92-93).

Sin embargo, el novedoso acercamiento al tema propuesto por Cruz Lara deja abierta la puerta para estudios futuros de mayor profundidad - que tendrían que acompañarse de fotografías de época para que el lector tenga un contexto más claro de la trama histórica-, que enriquezcan la visión del México porfirista para el lector novel y fortalezcan la discusión sobre la posición nacionalista de los arqueólogos de principios del siglo Xx o acerca de las actividades que realizaban en el Club Arqueológico.

Por ahora los invito a leer este texto, cuya temática invita a la reflexión.

\section{Referencias}

Cruz Lara Silva, Adriana

2011 El nacionalismo como eje interpretativo del objeto prehispánico. La restauración de tres urnas zapotecas de los siglos XIX y XX, México, INAH (Conservación y Restauración del Patrimonio. Serie Fundamentos). 